\title{
Gestión de los factores de riesgo operacional en las instituciones del sistema financiero popular y solidario
}

Management of operational risk factors in the institutions of the popular and solidarity financial system

\author{
Karina Johanna Iza López. ${ }^{1}$, Darwin Vladimir Rivera Piñaloza. ${ }^{2}$, \& Oscar Paúl \\ Tanqueño Colcha. ${ }^{3}$
}

Recibido: 09-05-2021 / Revisado: 20-05-2021 /Aceptado: 13-06-2021/ Publicado: 05-07-2021

\begin{abstract}
. DOI: https://doi.org/10.33262/concienciadigital.v4i3.1765

Introduction: in the 21 st century there are some risks to which the institutions of the Popular and Solidarity Financial Sector are exposed, such as the Savings and Credit Cooperatives COAC's, one of them is the operational risk that must be managed an efficient way to minimize its nascent impact of four factors that make it up: people, processes, information and communication technology and external events. Objective. Determine the degree of management of operational risk factors in the institutions of the Popular and Solidarity Financial System based on Resolution No. SEPS-IGT-2018-0279. Methodology. The approach, design, type and level of research was mixed considering qualitative and quantitative elements, not experimental, descriptive and documentary. The population was made up of six COACs belonging to segment 1 of the city of Guaranda, from which no sample was drawn due to a reduced group. To collect the information, the technique and the survey and questionnaire instrument were used in that

\footnotetext{
${ }^{1}$ Universidad Estatal de Bolívar, Facultad de Ciencias Administrativas, Gestión Empresarial e Informática, Carrera de Contabilidad y Auditoría, Guaranda, Ecuador, kiza@ueb.edu.ec, https://orcid.org/0000-00025111-8792

${ }^{2}$ Universidad Estatal de Bolívar, Facultad de Ciencias Administrativas, Gestión Empresarial e Informática, Carrera de Contabilidad y Auditoría, Guaranda, Ecuador, vrivera@ueb.edu.ec, https://orcid.org/00000002-5695-9726

${ }^{3}$ Universidad Estatal de Bolívar, Facultad de Ciencias Administrativas, Gestión Empresarial e Informática, Carrera de Contabilidad y Auditoría, Guaranda, Ecuador, otanqueno@ueb.edu.ec, https://orcid.org/00000002-5718-335X
} 
order. The questionnaire was subjected to a validation process developed by ten experts in the field to determine with consistency and reliability. Results. The investigation revealed that the COAC"s are managing operational risk factors based on Resolution No. SEPS-IGT-2018-0279. Organizations are managing the four operational risk factors according to the tools available to them. Conclusion. The COACs are managing the operational risk based on the regulations issued by the Regulatory Bodies.

Keywords: savings and credit cooperatives, operational risk, people, processes, information and communication technology, external events.

\section{Resumen.}

Introducción: en el siglo XXI algunos son los riesgos a los que están expuestas las instituciones del Sector Financiero Popular y Solidario como es el caso particular las Cooperativas de Ahorro y Crédito COAC's, uno de ellos es el riego operacional que debe ser gestionado de una manera eficiente para minimizar su impacto naciente de cuatro factores que lo integran como son las personas, procesos, tecnología de la información y comunicación y los eventos externos. Objetivo. Determinar el grado de gestión de los factores de riesgo operacional en las instituciones del Sistema Financiero Popular y Solidario tomando como base la Resolución No. SEPS-IGT-2018-0279. Metodología. El enfoque, diseño, tipo y nivel de investigación fue mixto por considerar elementos cualitativos y cuantitativos, no experimental, descriptivo y documental. La población estuvo integrada por seis COAC's pertenecientes al segmento 1 de la ciudad de Guaranda de la cual no se extrajo muestra alguna debido a que un grupo reducido. Para la recolección de la información se empleó la técnica y el instrumento encuesta y cuestionario en ese orden. El cuestionario fue sometido a un proceso de validación desarrollado por diez expertos en la materia para determinar con consistencia y confiabilidad. Resultados. De la investigación se desprendió que las COAC“'s si están gestionando los factores de riesgo operacional en base la Resolución No. SEPS-IGT2018-0279. Las organizaciones están gestionando los cuatro factores de riesgo operacional conforme las herramientas que tienen a su alcance. Conclusión. Las COAC's están administrando el riesgo operacional en base a la normativa emitida por los Órganos Reguladores.

Palabras clave: cooperativas de ahorro y crédito, riesgo operacional, personas, procesos, tecnología de la información y comunicación, eventos externos.

\section{Introducción.}

El sistema financiero del Ecuador está integrado por instituciones financieras como bancos, cooperativas de ahorro y crédito, mutualistas, sociedades financieras, entre otras que está bajo la regulación de la Superintendencia de Bancos (SB) y la Superintendencia de Economía Popular y Solidaria (SEPS). En la actualidad las empresas sean o no instituciones financieras están expuestas a distintos riesgos financieros, uno de ellos el 
riesgo operacional, el mismo que debe ser gestionado de manera apropiada puesto que puede causar distintos tipos de pérdidas como económicas, financieras y en la imagen institucional que puede ser producto de los cuatro factores que lo integran como son: 1) las personas, 2) procesos, 3) TIC's; y, 4) sucesos externos, este último está fuera del control de la empresa. Las COAC's que están bajo la regulación de la Superintendencia de Economía Popular y Solidaria (SEPS) para la gestión del riesgo operacional disponen de la Resolución No. SEPS-IGT-2018-0279 que es la norma responsable de identificar, valorar y controlar este riesgo, pero también el riesgo legal.

En este contexto, el riesgo operacional está inmerso en todas las tareas realizadas, decisiones tomadas, estrategias implementadas y tecnología empleada por las organizaciones lo cual representa una razón para instaurar medidas de protección para minimizar su probable impacto (Bravo Bravo \& Carrillo Lanas , 2020). La gestión realizada por las COAC's a grado global es menos dura que la ejercida por los bancos. El Ecuador no está alejado de esta ineludible realidad pues las instituciones financieras cuentan con el marco jurídico, pero en algunos casos la capacidad financiera los limita y no pueden desarrollar o adquirir herramientas de gestión empresarial alineado al riesgo operacional. En el país las COAC's están segmentadas de acuerdo a los señalado por el artículo 444 del Código Orgánico Monetario y Financiero en el que se indica que estas organizaciones serán ubicadas en los segmentos dados en el rango del 1-5 por la Junta de Política y Regulación Monetaria y Financiera.

En este orden de ideas, el presente artículo científico se plantea como problema de investigación ¿Las COAC’s están gestionando los factores de riesgo operacional de acuerdo al marco jurídico vigente? En tal sentido, el objetivo del estudio es determinar el grado de gestión de los factores de riesgo operacional en las instituciones del sistema financiero popular y solidario tomando como base la Resolución No. SEPS-IGT-20180279 .

Los riesgos a los que están expuestas las COAC’s son: el de crédito, liquidez, operacional, mercado, legal, y el de imagen institucional (reputacional). Entendiendo, que el término de riesgo se traduce en una contingencia, probabilidad o proximidad de que se origine un perjuicio, daño, pérdida u otro infortunio que puede afectar a una persona natural o jurídica (Reynaldo Argüelles, Guardado Lacaba, Sorhegui Ortega, \& Rojas de la Cruz, 2019). Por otro lado, en lo que respecta a las instituciones del sistema financiero el riesgo es un término empleado para denotar peligro causante de pérdidas de distinta índole interfiriendo en el logro de los propósitos estratégicos (Salinas Vásquez \& Lojano Cabrera, 2019).

Frente a la exposición de los riesgos financieros los Órganos Reguladores de las instituciones financieras a grado global han determinado que cada una de estas deben gestionarlos mediante acciones que ayuden a lograr el resultado esperado alineado a un enfoque estructurado para minimizar su impacto (Toro Díaz, Carmona Grajales, \& Tabares Torres, 2018; Lizarzaburu Bolaños, Barriga , Burneo, \& Noriega, 2019).La gestión de riesgos financieros también es entendida como un cumulo de estrategias, 
esfuerzos, compromisos desarrollados por el grupo social que integra la estructura organizacional dirigidos a minimizar el impacto que pueden generar (Alonso C. J. C. y Berggrun, 2015). El propósito de la gestión de riesgos es garantizar que no se produzcan pérdidas financieras, económicas o de imagen mediante prácticas empresariales orientadas a minimizar el impacto de los riesgos financieros, puesto que no existe el riesgo cero. En esta gestión de riegos está incluido el riesgo operacional que limita la continuidad del negocios si no se toman acciones oportunas para minimizar su impacto por parte de las empresas y de los organismos externos de regulación mediante la incorporación de juicios de identificación, valoración y vigilancia en el marco regulatorio (Bedoya Londoño, 2017). En consecuencia, la gestión de riesgos en implementar un sinnúmero de acciones encaminadas a la identificación (tipo de riesgos), medición (impacto-ocurrencia), control (monitoreo, seguimiento, acompañamiento) para proteger a las instituciones y de los grupos de interés relacionados con la misma de manera directa o indirecta (Orden Olasagasti, 2015).

El riesgo operacional es un riesgo financiero que representa probabilidad de pérdida dado en factores como: 1) personas o talento humano, este factor está alineado a los errores, fallas, incumplimientos de los procesos de administración del grupo social de la empresa como los de incorporación (selección y admisión de empleo), permanencia (valoración de desempeño, plan de desarrollo de carrera, entre otros), disolución laboral (planificación de la salida del personal), 2) procesos, representan los procesos y procedimientos críticos que desarrollan las empresas de acuerdo a la estructura organizacional organizado e acuerdo a los grados jerárquicos gobernante, habilitante u operacional; 3) tecnología de la información y la comunicación (TIC’s), está dado por la vulneración de herramientas de tratamiento de información, accesos no permitidos, paralización de los programas informáticos, daño a la calidad de información, entre otros, 4) incidentes externos, son las posibles pérdidas resultantes de sucesos externos como desastres naturales, atentados, actos ilícitos, vandalismo, entre otros (García Regal \& Seminario Olortigue, 2018; León, Scacco, \& Galiano, 2019).

El sistema financiero popular y solidario es uno de los sectores más importantes de la economía puesto que está integrado principalmente por cooperativas de ahorro y crédito, mismas que se dedican a la intermediación financiera y adecuaron sus estatutos de acuerdo a los requerimientos efectuados por los Órganos Reguladores de acuerdo a disposición transitoria primera de la Ley Orgánica de Economía Popular y Solidaria (LOEPS) en su momento (Ortega Pereira, Borja Borja, Aguilar Rodríguez, \& Moreno Cazar, 2018). Entendiéndose como cooperativa de ahorro y crédito a aquellas organizaciones en las que se han unido un grupo de personas bajo el principio de adhesión abierta y voluntaria para satisfacer sus necesidades económicas, sociales y culturales con una participación netamente democrática (Coraggio, 2011). 


\begin{tabular}{|c|c|}
\hline 1 & Mayor a $80^{\prime} 000.000$ \\
\hline 2 & Mayor a $20^{\prime} 000.000$ hasta $80^{\prime} 000.000$ \\
\hline 3 & Mayor a $5^{\prime} 000.000$ hasta $20^{\prime} 000.000$ \\
\hline 4 & Mayor a $1^{\prime} 000.000$ hasta $5^{\prime} 000.000$ \\
\hline 5 & $\begin{array}{l}\text { Hasta 1'000.000 Cajas de Ahorro, Bancos Comunes y } \\
\text { Cajas comunales. }\end{array}$ \\
\hline
\end{tabular}

Tabla 1. Segmentación de las COAC's por el valor de activos

Fuente: (Junta de Política y Regulación Monetaria y Financiera, 2015).

Una de las diligencias de las COAC's ecuatorianas es la gestión de los disantos riesgos financieros a los que están expuestas conforme la Resolución No. 128-2015-F como aquellos relacionados con 1) cartera de crédito, 2) tasas de interés, 3) indicadores de liquidez, 4) incumplimiento de leyes, resoluciones, manuales, reglamentos, políticas, 5) talento humano, procesos, procedimientos, tecnología, agentes externos; y, 6) imagen institucional.

\section{Metodología.}

Para la investigación se empleó un enfoque de investigación mixto, cualitativo porque se hizo el análisis de datos no numéricos, cuantitativos porque se procesó información numérica desprendida de la aplicación de técnicas e instrumentos de recolección de datos. El diseño fue no experimental por que se analizó a las COAC's en su estado natural sin ningún tipo de interferencia. El alcance fue descriptivo porque se detalló de manera minuciosa los cuatro factores que integran el riesgo operacional. La población del estudio estuvo integrada por seis COAC's del Sistema Financiero Popular y Solidario ubicadas en la ciudad de Guaranda pertenecientes al segmento 1, no se extrajo una muestra por ser una población pequeña. Las técnicas empleadas para a recolección de la información fueron la encuesta y el análisis documental y los instrumentos el cuestionario y la ficha bibliográfica. El cuestionario se lo estructuró con preguntas dicotómicas y polinómicas mismo que fue validado en inherencia a su consistencia y confiabilidad mediante el soporte de 10 expertos y el coeficiente Alpa de Cronbach, obteniendo un factor de 0, 91 denotando excelencia en los parámetros medidos y para el análisis documental se tomó como referencia diversa bibliografía compuesta por libros, artículos científicos y resoluciones emitidas por los Órganos de Control de este tipo de instituciones financieras. La validez del cuestionario y el procesamiento de la información se lo hizo con el soporte del programa SPSS versión 20.0. 


\begin{tabular}{|c|c|c|}
\hline No. & RUC & Razón Social \\
\hline 1 & 1891710328001 & Cooperativa de Ahorro y Crédito Chibuleo Ltda. \\
\hline 2 & 1790866084001 & $\begin{array}{l}\text { Cooperativa de Ahorro y Crédito Policía Nacional } \\
\text { Ltda. }\end{array}$ \\
\hline 3 & 1890141877001 & $\begin{array}{l}\text { Cooperativa de Ahorro y Crédito Mushuc Runa } \\
\text { Ltda. }\end{array}$ \\
\hline 4 & 1890037646001 & Cooperativa de Ahorro y Crédito el Sagrario Ltda. \\
\hline 5 & 290003288001 & Cooperativa de Ahorro y Crédito San José Ltda. \\
\hline 6 & 1891709591001 & Cooperativa de Ahorro y Crédito Ambato Ltda. \\
\hline
\end{tabular}

Tabla 2. Coac's del sector financiero popular y solidario segmento 1

Fuente: (Superintedencia de Economía Popular y Solidaria, 2020)

\section{Resultados.}

\section{Gestión del riesgo operacional}

Los gestores de las COAC's manifestaron en su totalidad que si cuentan con una unidad de riesgos y que la misma es el área encargada de la gestión de los riesgos a los que están expuestas mediante la práctica de conocimientos de la persona responsable catalogado según normativa como Oficial de Riesgos, el cual desarrolla acciones como: 1) la identificación de los riesgos mediante la utilización de una matriz de riesgos y otras herramientas, 2) medición del riesgo, con la valoración de la pérdida dada y el posible daño que podría ocasionar; y, 3) control del riesgo, mediante la verificación del grado de respeto a las medidas de protección establecidas para minimizar el impacto. Por otro lado, en referencia al Comité Integra de Riesgos (CAIR) las organizaciones lo han definido conforme la Resolución No. 128-F-2015 que fue emitida de manera exclusiva para las instituciones financieras que están bajo el control de la Superintendencia de Economía Popular y Solidaria (SEPS) para la administración integral de riesgos dirigido al tratamiento de todos los riesgos incluido el operacional. En relación al riesgo al que más expuestas han estado por el número de sucesos dados, el $83 \%$ señalaron que al riesgo operacional y el $17 \%$ al riesgo de crédito, este último dado por incumplimientos en los pagos de operaciones crediticias adquiridas.

El 50\% de los encuestados indicaron que el factor que más incidencia tiene en el riesgo operacional es el de personas, el 33\% indicaron que los eventos externos y el 17\% los procesos. También indicaron que cuentan con un plan de continuidad del negocio (PCN) que es el documento logístico en el cual consta una serie de acciones para reaccionar en tres escenarios antes, durante y después de la ocurrencia de un evento inesperado sea por concepto de recuperación o restauración de los procesos críticos. El personal de la empresa de las matrices y agencias de las COAC's están siendo permanentemente instruidos en temas asociados al riesgo operacional incluido en temas de simulaciones en 
tiempo real frente a determinado suceso inesperado esto para crear una cultura de riesgos (Ve tabla 3).

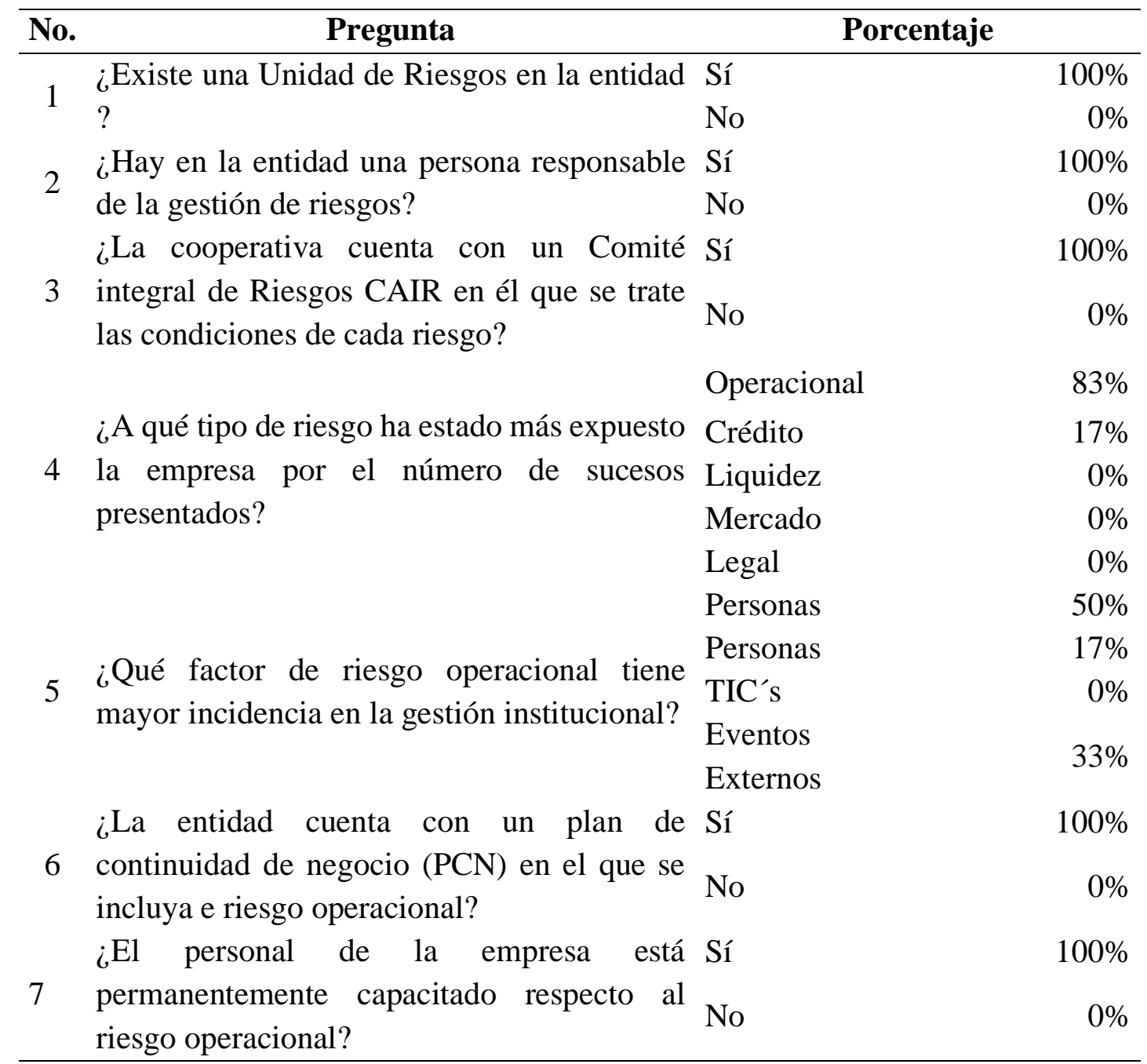

Tabla 3. Gestión del riesgo operacional

Fuente: Elaboración propia

\section{Factor 1. Personas}

Referente a este factor las COAC's cuentan con procesos para la administración del recurso más importante como es el talento humano, por ejemplo, disponen con dos manuales: 1) manual de procesos de administración del talento humano, que es un documento técnico utilizado como herramienta de gestión de los procesos de incorporación, permanencia y de terminación de la relación contractual; y, 2) manual descriptor de cargos que es el documento en el que constan las funciones específicas de cada cargo, el perfil técnico, requisitos del puesto visto en las competencias necesarias, entre otros elementos. Las instituciones también indicaron en un $17 \%$ que, aunque disponen de un manual descriptor de cargos, pero este documento no está conforme la estructura organizacional, no existe una independencia de funciones ya que la segregación no es la apropiada, existe concentración incompatible de funciones lo cual daría lugar en 
especial al cometimiento de errores o actos ilícitos como el fraude, en cambio el 83\% señalaron que el manual descriptor de cargos está conforme la Resolución No. SEPSIGT-2018-0279.

Otro de los requerimientos de la resolución mencionada con antelación que requiere cumplan las Cooperativas de Ahorro y Crédito es disponer de una base de datos actualizada del personal de la empresa que registre datos básicos de los colaboradores, resultados de valoraciones de desempeño, beneficios sociales percibidos, número de horas extraordinarias y suplementarias, sanciones, llamados de atención entre otros aspectos, en este sentido el $33 \%$ señala que cuenta con una base de datos con algunos de los aspectos expuestos y que están trabajando en este tema y el 67\% indicaron que si cuentan con la base de datos misma que se ha traducido en una herramienta valiosa para la toma de decisiones respecto al grupo social que integran la empresa (Ve tabla 4).

\begin{tabular}{cllc}
\hline No. & \multicolumn{1}{c}{ Pregunta } & Porcentaje \\
\hline \multirow{2}{*}{1} & ¿La entidad cuenta con una manual de talento Sí & $100 \%$ \\
& humano ? & No & $0 \%$ \\
2 & ¿Existe una apropiada segregación de & Sí & $83 \%$ \\
& funciones? & No & $17 \%$ \\
\multirow{2}{*}{3} & ¿La entidad cuenta con una base de datos & Sí & $67 \%$ \\
& actualizada del talento humano? & No & $33 \%$ \\
\hline
\end{tabular}

Tabla 4. Factor personas

Fuente: Elaboración propia

\section{Factor 2. Procesos}

Los representantes de las COAC's aludieron en su totalidad que sí disponen del documento técnico de gestión de procesos que también se lo denominado como manual de procedimientos en el que consta lo que se debe hacer tomando como base al talento humano y lograr así que la empresa funcione de manera correcta, mismo que ha sido elaborado de la mano con el manual de descriptor de cargos, estructura organizacional, políticas, reglamentos, resoluciones, entre otra normativa legal vigente interna y externa. El $100 \%$ de los encuestados también indicaron que los procesos han sido definidos en base a políticas y que se han establecido metodologías para su diseño, actualización, aprobación, difusión e implementación. En relación a la interrogante asociada al portafolio de procesos el $100 \%$ indicaron que sí disponen de esta cartera de productos y que está estructurado de acuerdo a las líneas de negocios de la organización y contiene datos básicos como tipo de proceso de acuerdo a la estructura organizacional, valoración del proceso respecto a la criticidad, responsable, entre otros datos (Ve tabla 5).

\begin{tabular}{cclc}
\hline No. & Pregunta & \multicolumn{2}{c}{ Porcentaje } \\
\hline \multirow{2}{*}{1} & ¿La entidad cuenta con una manual de & Sí & $100 \%$ \\
& administración de procesos? & No & $0 \%$ \\
\hline
\end{tabular}


¿Los procesos están definidos conforme los Sí $\quad 100 \%$

2 grados jerárquicos gobernante, habilitante $\mathrm{y}$ operacional?

No $\quad 0 \%$

3 ¿La entidad tiene definido el portafolio de Sí $\quad 100 \%$ procesos?

No

$0 \%$

Tabla 5. Factor procesos

Fuente: Elaboración propia

\section{Factor 3. Tecnología de la Información y Comunicación}

Para la gestión de la Tecnología de la Información y Comunicación (TIC's) las instituciones financieras (IFI's) deben contar con una estructura formal. En el caso de las COAC's de segmento 1 objeto de análisis el $100 \%$ cuentan con una unidad y un comité de Tecnología de la Información y Comunicación debidamente formalizado al interior de estas, instancias integradas por el mimo grupo social que las integran con el afán de garantizar la continuidad del negocio esto lo hicieron una vez que entró en vigencia la Resolución No. SEPS-IGT-2018-0279 dentro de los plazos establecidos para el efecto. En consecuencia, disponen de políticas, procesos, procedimientos para la gestión tecnológica debidamente diseñadas, actualizadas, aprobadas, difundidas e implementadas. En consecuencia, para una adecuada gestión de las TIC's las COAC's cuentan con un plan de contingencia que es un documento que integra una serie de procesos para hacer frente a los eventos inesperados en escenarios de reacción y recuperación. Tal es así que este documento es implementado por estas organizaciones cuando se da el suceso no planeado y está en constante actualización para asegurar medidas de protección (Ve tabla 6).

\begin{tabular}{clllc}
\hline No. & \multicolumn{1}{c}{ Pregunta } & Porcentaje \\
\hline 1 & $\begin{array}{l}\text { ¿La entidad cuenta estructura de gestión Sí } \\
\text { tecnológica? }\end{array}$ & No & $0 \%$ \\
2 & $\begin{array}{l}\text { ¿Estas definidas políticas, procesos, Sí } \\
\text { procedimientos para la gestión tecnológica? No }\end{array}$ & $100 \%$ \\
& $\begin{array}{l}\text { ¿La entidad tiene definido un plan de Sí } \\
\text { contingencia para garantizar la continuidad del No } \\
\text { negocio? }\end{array}$ & $67 \%$ \\
\end{tabular}

Tabla 6. Factor TIC's

Fuente: Elaboración propia

\section{Factor 4. Eventos Externos}

Para una buena gestión del riesgo operacional las entidades deben tener presente que las pérdidas también pueden originarse de variables que están fuera de su control como son los eventos externos o también denominados agentes externos. A este respecto las COAC's en un $50 \%$ indicaron que a los incidentes que más expuestos han estado 
relacionados a los fraudes externos que son actos ilícitos cometidos por personas u organizaciones ajenas a estas, el $25 \%$ el vandalismo entendiendo que este una serie de acciones con el propósito de destruir, en el caso particular de estas organizaciones ha sufrido daños a la infraestructura física, cajeros automáticos que son bienes que están más al alcance del público y el $25 \%$ mencionaron que hasta el momento no han sido objeto de ningún evento externo; sin embargo, no descartan la probabilidad de ocurrencia por ello están trabajando arduamente en este tema mediante el establecimiento de controles de acuerdo a su realidad y capacidad financiera para garantizar medidas de seguridad (Ve tabla 7).

\begin{tabular}{|c|c|c|c|}
\hline No. & Pregunta & Po & \\
\hline \multirow{4}{*}{1} & \multirow{4}{*}{$\begin{array}{l}\text { ¿Cuál ha sido el evento } \\
\text { externo al que mayor expuesta } \\
\text { esta la entidad? }\end{array}$} & Desastres naturales & $0 \%$ \\
\hline & & Fraudes externos & $50 \%$ \\
\hline & & Vandalismo & $25 \%$ \\
\hline & & Ninguno & $25 \%$ \\
\hline
\end{tabular}

Tabla 7. Factor eventos externos

Fuente: elaboración propia

\section{Conclusiones.}

- Las organizaciones tomadas para el análisis han cumplido en gran parte de la Resolución No. SEPS-IGT-2018-0279 puesto que los factores de riesgo operacional han sido gestionados de manera apropiada. Esto se vislumbra en que su totalidad disponen de una unidad de riesgos y un comité de administración integral de riesgos (CAIR) que está integrado por el Oficial de Riesgos y autoridades desprendidos del grado jerárquico Gobernante. El riesgo al que más expuesto han estado las entidades es el riesgo operacional y que los factores que más inciden en este riesgo son las personas, eventos externos y procesos respecto a las TIC's menor ha sido el número de sucesos. La entidad cuenta con un plan de continuidad del negocio garantizando así la funcionalidad en el caso presencia de casos fortuitos y su desarrollo es respaldado por contar con personal calificado lo que representa también un aliciente para las COAC's.

- Las COAc's cuentan con herramientas de gestión del personal como un manual de talento humano que incluye procesos de admisión y empleo, pero no basta puesto que, aunque en la mayor parte de estas empresas estén debidamente segregadas las funciones de acuerdo a ciertos parámetros, existen organizaciones en las cuáles están focalizadas funciones incompatibles y no están bajo ciertas medidas constantes en la Resolución No. SEPS-IGT-2018-0279. Por otro lado, respecto a si disponen una base de datos del personal actualizada mencionaron que sí disponen de información del personal, pero en algunos casos no actualizada porque hasta el momento la está depurando de acuerdo a lo estipulado en la norma.

- Las instituciones financieras consultadas indican que si cuentan con instrumento técnico para la gestión de procesos como es un manual que integra procesos 
definidos conforme los grados jerárquicos de la estructura organizacional y así también tienen definido el portafolio de procesos, lo cual denota que este factor de riesgo está fortalecido por las acciones implementadas.

- Referente al factor tecnología de la información y comunicación (TIC's) dispone de una adecuada estructura de gestión integrada por una unidad y un comité los cuales tiene definidos sus funciones de acuerdo a lo que señala la norma y la realidad institucional. Una de las fortalezas que presentan todas las organizaciones es que tienen definido en su totalidad procesos, procedimientos y políticas. Por otro lado, una debilidad es que algunas de las Cooperativas no disponen de un plan de contingencia para las TIC'S lo que podría instaurarse en un factor de fracaso.

- El evento externo al que más expuesto han estado las organizaciones en los últimos tiempos son los fraudes externos dado por actos ilícitos y vandalismo destrucción de infraestructura física y tecnológica está última en cajeros automáticos cuyo impacto no ha sido alto, pero a partir de ello han tomado las medidas necesarias para contrarrestar esta situación.

\section{Referencias bibliográficas.}

Alonso C. J. C. y Berggrun, P. (2015). Introducción al análisis de riesgo financiero (3a. ed.). Bogotá: Ecoe Ediciones.

Bedoya Londoño, D. A. (2017). Análisis de la evolución del uso de las cuentas contables de riesgo operativo en las entidades financieras en Colombia. Espacios, 39(9), 521.

Bravo Bravo , P. A., \& Carrillo Lanas , E. X. (2020). Modelo de gestión del riesgo operativo para el control de la gestión financiera en el Instituto Superior Tecnológico Para el Desarrollo ISPADE. Tesis [Maestría]. Universidad Andina Simón Bolívar, Quito.

Coraggio, J. L. (2011). Economía Social y Solidaria: El trabajo antes que el capital. Quito: Ediciones AbyaYala.

García Regal, R. F., \& Seminario Olortigue, R. M. (2018). Gestión de riesgo operacional y su relación con la utilidad bruta de la Financiera Manco Inca (Lima, 20132017) . Tesis [Maestría]. Universidad Católica Sedes Sapientiae, LIma.

Junta de Política y Regulación Monetaria y Financiera. (2015). Resolución No. 038-2015F. Quito-Ecuador. Obtenido de https://n9.cl/duus

Junta de Política y Regulación Monetaria y Financiera. (2015). Resolución No. 128-2015F. Quito-Ecuador. Obtenido de http://www.seps.gob. ec/documents/20181/25522/Resol128.pdf/5c74f247-0de4-4862-

b5fbc89ff62817d7 
León, R. G., Scacco, E. B., \& Galiano, N. E. (2019). Identificación de factores de riesgo operativo en el sector metalmecánico manufacturero. Espacios, 4(20), 23-37. doi:https://n9.cl/i1t2u

Lizarzaburu Bolaños, E. R., Barriga , G., Burneo, K., \& Noriega, E. (2019). Gestión Integral de Riesgos y Antisoborno: Un enfoque operacional desde la perspectiva iso $31000 \mathrm{e}$ iso 37001. Universidad \& Empresa, 21(36), 79-118. doi:https://doi.org/10.12804/revistas.urosario.edu.co/empresa/a.6089

Orden Olasagasti, O. D. (2015). Gestión del riesgo y mercados financieros. Madrid: Delta Publicaciones.

Ortega Pereira, J. R., Borja Borja, M., Aguilar Rodríguez, I. E., \& Moreno Cazar, W. M. (2018). Sistema Financiero Popular y Solidario y su evolución desde la implementación de la nueva Ley Orgánica de Economía Popular y Solidaria. Cooperativismo y Desarrollo, 5(113), 1-17.

Reynaldo Argüelles, C. L., Guardado Lacaba, R. M., Sorhegui Ortega, R. A., \& Rojas de la Cruz, R. (2019). Importancia de la gestión de riesgos para el desarrollo local. caso de estudio consejo popular caribe, Cuba. Ecociencia, 6(5), 1-23. Obtenido de https://n9.cl/gssyh

Salinas Vásquez, J. V., \& Lojano Cabrera, V. A. (2019). Análisis de los factores que inciden en la gestión de riesgos financieros en las cooperativas de ahorro y crédito del segmento cinco, Cuenca 2018. Observatorio de la Economía Latinoamericana, 1-15. Obtenido de https://www.eumed.net/rev/oel/2019/02/gestion-riesgos-financieros.html

Superintedencia de Economía Popular y Solidaria. (2020). Segmentación 2020 del sector Financiero Popular y Solidario. Obtenido de https://n9.cl/2rhma

Toro Díaz, J., Carmona Grajales, J., \& Tabares Torres, M. (2018). Riesgo financiero en empresas espñolas. Empresarial, 46(1), 52 - 61 .

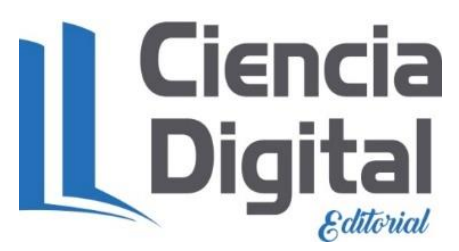




\section{PARA CITAR EL ARTÍCULO INDEXADO.}

Iza López, K. J., Rivera Piñaloza, D. V., \& Tanqueño Colcha, O. P. (2021). Gestión de los factores de riesgo operacional en las instituciones del sistema financiero popular y solidario.

ConcienciaDigital,

$4(3)$,

81-93.

https://doi.org/10.33262/concienciadigital.v4i3.1765

\section{Ciencia \\ LDigital}

El artículo que se publica es de exclusiva responsabilidad de los autores y no necesariamente reflejan el pensamiento de la Revista Conciencia Digital.

El artículo queda en propiedad de la revista y, por tanto, su publicación parcial y/o total en otro medio tiene que ser autorizado por el director de la Revista Conciencia Digital.

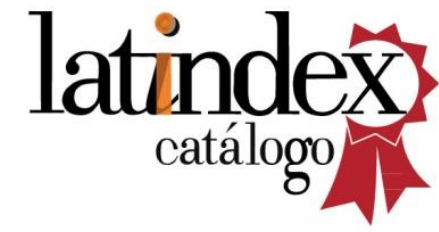

